\title{
ACID CARBOXYPEPTIDASE DEFICIENCY IN GALACTOSIALIDOSIS
}

\author{
Kohji ItoH, ${ }^{1}$ Nobuaki TakiYama, ${ }^{2}$ Yoshiro Nagao, ${ }^{1}$ Akihiro Oshima, ${ }^{1}$ \\ Hitoshi SakUraba, ${ }^{1}$ Michel Potier, ${ }^{3}$ and Yoshiyuki Suzuki ${ }^{1}$ \\ ${ }^{1}$ Department of Clinical Genetics, The Tokyo Metropolitan Institute of Medical Science, \\ Honkomagome, Bônkyo-ku, Tokyo 113, Japan \\ ${ }^{2}$ Department of Pediatrics, Saiseikai Utsunomiya Hospital, \\ Utsumoniya 320, Japan \\ ${ }^{3}$ Service de Genetique Medicale, Hôpital Saint-Justine, \\ Montreal, Quebec, Canada H3T 1C5
}

\begin{abstract}
Summary Carboxypeptidase activity with an optimal pH at 5.7 was found to be deficient in cultured lymphoblastoid cells and skin fibroblasts from 16 galactosialidosis patients of Japanese origin. The amounts of residual enzyme activities did not correlate with clinical phenotypes (early infantile and juvenile/adult). Four parents of the patients from different families showed enzyme activities at an intermediate level between the patients and normal controls. It was concluded that this enzyme deficiency is closely connected to the genetic defect of "protective protein." Further characterization with various protease inhibitors indicated that the enzyme deficient in galactosialidosis cells is a serine carboxypeptidase with histidine and cysteine residues at or near the active site.
\end{abstract}

Key Words galactosialidosis, carboxypeptidase, protective protein, $\beta$-galactosidase, neuraminidase

\section{INTRODUCTION}

Galactosialidosis is a genetic metabolic disease caused by a deficiency of "protective protein" which stabilizes lysosomal $\beta$-galactosidase [EC 3.2.1.23] (d'Azzo et al., 1982; Hoogeveen et al., 1983) and activates lysosomal neuraminidase [EC 3.2.1.18] (Verheijen et al., 1985; Nanba et al., 1987). More than 50 cases of galactosialidosis have been reported and about $70 \%$ of them are of Japanese origin (Suzuki et al., 1984, 1988). Most patients developed loss of vision as an initial symptom at ages 10-15 years, followed by neurological abnormalities, such as action myoclonus, cerebellar ataxia, skeletal dysplasia, cherry-red spots, and angiokeratoma (juvenile/adult form) (Suzuki et al., 1988). Clinically severe cases have also been

Received December 19, 1990; Accepted February 6, 1991. 
reported with edema, ascites, skeletal dysplasia, and cherry-red spots, oscurring in early infancy (early infantile form) (Suzuki et al., 1988). However, the correlation between the clinical phenotypes and genetic/biochemical data has not been clear.

The cDNAs encoding human and murine "protective protein" have been cloned, and the deduced amino acid sequences were found to possess homology to yeast carboxypeptidase Y and the KEX1 gene product (Galjart et al., 1988, 1990). Recently, the fibroblasts from three patients with late infantile form of galactosialidosis were found to be deficient in carboxypeptidase activity (Tranchemontagne et al., 1990). The present study demonstrated that the acid carboxypeptidase activity was markedly reduced in lymphoblastoid cells as well as in fibroblasts from various clinical forms of galactosialidosis patients, and further characterization of this enzyme activity was performed.

\section{MATERIALS AND METHODS}

Cells. The cells for this study were obtained from control subjects, galactosialidosis patients, and some of their parents. Fibroblasts were cultured in Ham's F-10 medium supplemented with $10 \%$ fetal calf serum and antibiotics. Lymphoblastoid cell lines were established as described by Anderson and Gusella (1984). The diagnosis of galactosialidosis was confirmed by clinical manifestations and assays of lysosomal enzymes in mixed leukocytes and/or fibroblasts (Sakuraba et al., 1982). The clinical phenotypes were classified as early infantile form in 2 unrelated patients and juvenile/adult form in 14 patients from 9 families (Suzuki et al., 1988).

Neuraminidase and $\beta$-galactosidase assays. Activities of neuraminidase and $\beta$-galactosidase were assayed as described previously, using 4-methylumbelliferyl glycosides as substrates (Sakuraba et al., 1982). Protein determination was performed according to the method of Bradford (1976) using bovine serum albumin as standard.

Carboxypeptidase assay. Carboxypeptidase activity was measured in skin fibroblasts and lymphoblastoid cells by a modified method of Stevens et al. (1986), using $N$-CBZ-L-phenylalanyl-L-leucine ( $N$-CBZ-Phe-Leu) as substrate. The cell pellets were suspended in distilled water, and homogenized by sonication for 10 sec. The cell homogenate $(5-8 \mu \mathrm{g}$ protein for fibroblasts, and $50-100 \mu \mathrm{g}$ protein for lymphoblastoid cells) was incubated at $25^{\circ} \mathrm{C}$ in $50 \mathrm{~mm}$ sodium acetate buffer, pH 5.7 , containing $0.75 \mathrm{~mm} N$-CBZ-Phe-Leu in a total volume of $0.1 \mathrm{ml}$. After $30 \mathrm{~min}, 0.1 \mathrm{ml}$ of distilled water was added, and the reaction was stopped by boiling for $3 \mathrm{~min}$. The released L-leucine was determined by the method of Stevens et al. (1986).

Characterization of acid carboxypeptidase. The following reagents were used for characterization of the carboxypeptidase activity; active site serine reagent 
phenylmethylsulfonyl fluoride (PMSF) (Sigma, St Louis, U.S.A.); histidine reagents chloromethylketone derivatives of $N$-CBZ-L-phenylalanine (ZPCK; Sigma) and $N$ - $\alpha$-tosyl-L-phenylalanine (TPCK; Nacalai Tesque, Kyoto); thiol reagent iodoacetamide (Sigma); metal-chelating agent EDTA (Wako Pure Chemicals, Osaka); thiol-serine protease inhibitor leupeptin, carboxyprotease inhibitor pepstatin A, and metalloprotease inhibitor phosphoramidon (Peptide Institute, Osaka). Each of these reagents was preincubated for $60 \mathrm{~min}$ at $25^{\circ} \mathrm{C}$ with a fibroblast homogenate $(5-8 \mu \mathrm{g}$ protein) in the presence of $25 \mathrm{~mm}$ sodium acetate buffer ( $\mathrm{pH} \mathrm{5.7)} \mathrm{in}$ a total volume of $50 \mu \mathrm{l}$. At the end of preincubation, $50 \mu \mathrm{l}$ of $1.5 \mathrm{~mm} N$-CBZ-PheLeu was added to the incubation mixture for carboxypeptidase assay as described above. The reagents in this experiment did not interfere with determination of the liberated L-leucine.

\section{RESULTS}

Acid carboxypeptidase activity in galactosialidosis cells

In the present study, we assayed and characterized the carboxypeptidase activity in fibroblasts and lymphoblastoid cells from galactosialidosis patients of different clinical forms (juvenile/adult form and early infantile form) and from some of their parents. This enzyme showed a maximal activity at pH 5.7 in control fibroblasts with a synthetic substrate ( $N$-CBZ-Phe-Leu), whereas it was almost completely deficient in the galactosialidosis cells (Fig. 1A). In lymphoblastoid cells, a broad
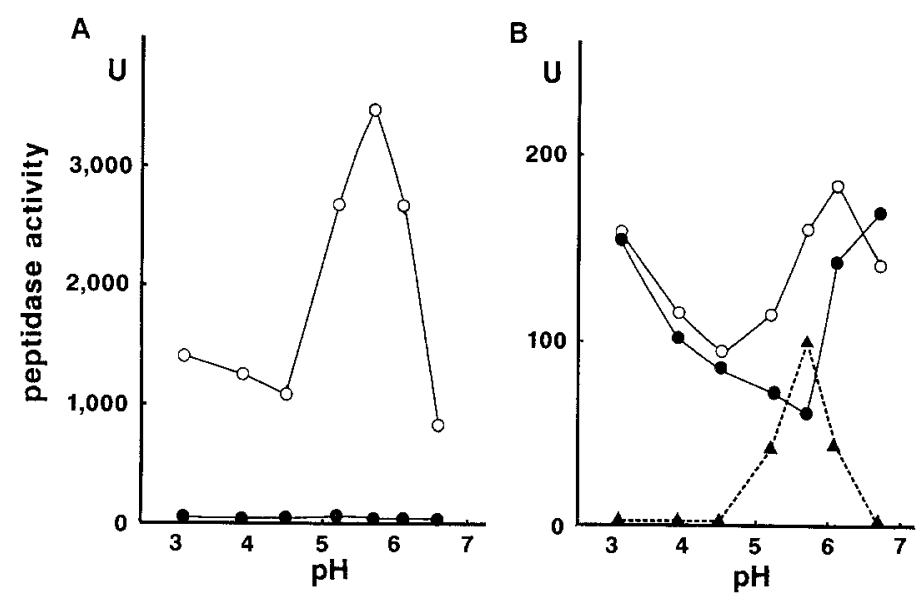

Fig. 1. pH-profiles of acid carboxypeptidase activity in fibroblasts and lymphoblastoid cells. Homogenates of fibroblasts and lymphoblastoid cells from a galactosialidosis patient and a control subject were prepared, and the carboxypeptidase activities were assayed as described in METHODS. Each value represents an average of duplicate determinations. Panel A, fibroblasts; panel B, lymphoblastoid cells. $O$, control subject; $\bullet$, galactosialidosis patient; $\boldsymbol{\Lambda}$, subtraction profile. 
$\mathrm{pH}$ profile was obtained, and a relatively low activity was observed in galactosialidosis cells at pH 5-6 (Fig. 1B). When a differential pH-activity curve was plotted after subtraction of the patient's value from the control value at each $\mathrm{pH}$, a single, sharp peak was elicited at $\mathrm{pH}$ 5.7. This result indicated that there are at least three peptidase activities in control lymphoblastoid cells, which have the optimal $\mathrm{pH}$ at 3.0, 5.7, and 6.8. It was concluded that only one of them, the carboxypeptidase with the optimum $\mathrm{pH}$ at 5.7 , was deficient in galactosialidosis cells.

Subsequently, we assayed the carboxypeptidase activity under the optimal assay $\mathrm{pH}$ as described above, together with $\beta$-galactosidase and neuraminidase activities, in fibroblasts and lymphoblastoid cells from galactosialidosis patients (Table 1). There was no significant difference in carboxypeptidase activities in. fibroblasts between the early-onset and late-onset forms of galactosialidosis patients ( $20 \%$ of the control mean for early-onset form, and $17 \%$ for late-onset form). The enzyme activity in lymphoblastoid cells was 24 and $23 \%$ of the control mean, respectively, for a patient of early infantile form and 11 patients of juvenile/adult form. The average enzyme activity was low in fibroblasts from 4 parents of 4 unrelated patients $(55 \%$ of the control mean; $p<0.05)$. Carboxypeptidase activity was normal in $\mathrm{G}_{M_{1} 1}$-gangliosidosis and sialidosis patients (Table 1).

Characterization of acid carboxypeptidase

For further characterization of the enzyme deficient in galactosialidosis cells,

Table 1. Enzyme activities in human cultured cells.

\begin{tabular}{lccc}
\hline & Neuraminidase & $\beta$-Galactosidase & Carboxypeptidase \\
\hline Fibroblasts & & & \\
Galactosialidosis & & & \\
1) Patients & & 125 & 813 \\
$\quad$ Early infantile & 15.6 & $42.4 \pm 24.6(7)$ & $659 \pm 362(7)$ \\
Juvenile/adult & $8.19 \pm 4.15(7)$ & $689 \pm 133(4)$ & $2,223 \pm 410(4)$ \\
2) Parents & $38.1 \pm 5.50(4)$ & $44.7 \pm 11.4(3)$ & $3,736 \pm 1,120(3)$ \\
GM & $65.6 \pm 29.3(3)$ & 372 & 2,500 \\
Sialidosis & 0.42 & $479 \pm 30.0(32)$ & $4,069 \pm 1,574(7)$ \\
Controls & $36.0 \pm 9.47(6)$ & & \\
Lymphoblastoid cells & & 7.32 & 75.8 \\
Galactosialidosis & 1.27 & $8.55 \pm 4.50(13)$ & $71.6 \pm 22.0(13)$ \\
Early infantile & $0.92 \pm 0.72(13)$ & 0.87 & 298 \\
Juvenile/adult & 8.50 & $54.1 \pm 17.5(9)$ & $322 \pm 157(9)$ \\
GM $_{1}$-gangliosidosis & $6.59 \pm 2.78(9)$ & & \\
Controls & & & \\
\hline
\end{tabular}

Enzyme activities are expressed as $\mathrm{nmol} / \mathrm{hr} / \mathrm{mg}$ protein [mean $\pm \mathrm{SD}(\mathrm{n})]$ 
Table 2. Effects of active site reagents and protease inhibitors on acid carboxypeptidase activity.

\begin{tabular}{lcc}
\hline Inhibitor & $\begin{array}{c}\text { Concentration } \\
(\mathrm{mm})\end{array}$ & $\begin{array}{c}\text { Enzyme activity } \\
(\% \text { of control mean })\end{array}$ \\
\hline None & - & 100 \\
PMSF & 1 & 12 \\
ZPCK & 5 & 2 \\
& 2.4 & 100 \\
TPCK & 12 & 35 \\
Iodoacetamide & 2.4 & 90 \\
& 12 & 64 \\
Leupeptin & 1 & 98 \\
& 5 & 45 \\
Pepstatin A & 0.5 & 93 \\
Phosphoramidon & 2.5 & 70 \\
& $1.5 \times 10^{-3}$ & 102 \\
EDTA & $15 \times 10^{-3}$ & 128 \\
& $1.8 \times 10^{-3}$ & 89 \\
& $18 \times 10^{-3}$ & 99 \\
& 5 & 118 \\
\end{tabular}

PMSF, phenylmethylsulfonyl fluoride; ZPCK, $N$-CBZ-L-phenylalanine chloromethylketone; TPCK, $N$ - $\alpha$-tosyl-L-phenylalanine chloromethylketone; EDTA, ethylenediaminetetraacetic acid.

we evaluated the effects of various reagents acting on enzyme proteins. As shown in Table 2, PMSF, which binds to a serine residue, inhibited the carboxypeptidase activity. Relatively high concentrations of histidine reagents ZPCK and TPCK, a thiol-serine protease inhibitor leupeptin, and a cysteine reagent iodoacetamide also partially inhibited the enzyme activity. On the contrary, acid protease inhibitor pepstatin A, and metallo-protease inhibitors EDTA and phosphoramidon did not show any inhibitory effects on the activity. These results indicated that the acid carboxypeptidase deficient in galactosialidosis cells is a serine carboxypeptidase with histidine and cysteine residues at or near the active site.

\section{DISCUSSION}

Clinical manifestations of galactosialidosis patients have been found variable, although most of the cases reported in Japan were classified as juvenile/adult form with slowly progressive central nervous system deteriorations (Suzuki et al., 1984, 1988). The early-onset form patients present with more generalized systemic mani- 
festations. The biochemical/genetic basis of variations in phenotypic expressions and ages of onset is not known at present.

The biological function of "protective protein" was first suggested to be associated with a protease activity by demonstration of a significant homology of deduced amino acid sequence to that of yeast carboxypeptidase Y (Galjart et al., 1988, 1990). A subsequent study (Tranchemontagne et al., 1990) demonstrated a specific deficiency of lysosomal carboxypeptidase ( $1.4 \%$ of the control mean) at $\mathrm{pH} 5.5$ in fibroblasts from galactosialidosis patients of late infantile form, possibly an intermediate form between early infantile and juvenile/adult forms. They also reported a carboxypeptidase $\mathrm{L}$ activity in the multi-enzymatic lysosomal complex of neuraminidase, $\beta$-galactosidase, and "protective protein" purified from human placenta, and suggested that the lysosomal carboxypeptidase activity was associated with "protective protein" (Potier et al., 1990). The sensitivity of this enzyme activity to various protease inhibitors was similar to that of carboxypeptidase $Y$, in which the active site residues were serine, histidine and cysteine (Tranchemontagne et al., 1990). However, direct evidence for identification of the carboxypeptidase as "protective protein" has not been presented.

A marked decrease of acid carboxypeptidase activity was found in lymphoblastoid cells as well as in fibroblasts from all galactosialidosis cases in this study. This result strongly suggests a close correlation of acid carboxypeptidase deficiency with "protective protein" defect in galactosialidosis. Tsuji et al. (1983) reported that the lysosomal neuraminidase was significantly low $(70 \%$ of the control level) in lymphocytes from the parents of galactosialidosis patients, and concluded that heterozygote detection was possible by neuraminidase assays. However, we could not detect any significant differences in neuraminidase activity as well as in $\beta$-galactosidase activity in parents' fibroblasts. On the other hand, carboxypeptidase activity in the parents was at an intermediate level between the patients and controls. This result also indicate that the low carboxypeptidase activity in the parents (obligate heterozygotes) results from a primary defect of this enzyme and represents a gene dosage effect of an autosomal recessive disease.

In our present assay system, no remarkable difference was found in carboxypeptidase activity between early infantile and juvenile/adult galactosialidosis cells, and we could not correlate clinical phenotypes with residual enzyme activities. The relation between the enzyme activity and the "protective" activity remains unknown. The former may have a direct effect on the latter in the lysosomal enzyme complex described above. This question will be solved by molecular analysis of these proteins, "protective protein"/carboxypeptidase, $\beta$-galactosidase, and neuraminidase in the future.

Acknowledgments The authors wish to thank Ms. Haruko Nakashima and Ms. Keiko Ishii for excellent technical assistance.

This work was supported by grants from the Ministry of Education, Science and Culture, and the Ministry of Health and Welfare of Japan. 


\section{REFERENCES}

Anderson, M.A. and Gusella, J.F. 1984. Use of cyclosporin A in establishing Epstein-Barr virustransformed human lymphoblastoid cell lines. In Vitro 20: 856-858.

Bradford, M.M. 1976. A rapid and sensitive method for the quantitation of microgram quantities of protein utilizing the principle of protein-dye binding. Anal. Biochem. 72: 248-254.

d'Azzo, A., Hoogeveen, A.T., Reuser, A.J.J., Robinson, D. and Galjaard, H. 1982. Molecular defect in combined $\beta$-galactosidase and neuraminidase deficiency in man. Proc. Natl. Acad. Sci. U.S.A. 79: 4535-4539.

Galjart, N.J., Gillemans, N., Harris, A., van der Horst, G.T.J., Verheijen, F.W., Galjaard, H. and d'Azzo, A. 1988. Expression of cDNA encoding the human "protective protein" associated with lysosomal $\beta$-galactosidase and neuraminidase: Homology to yeast proteases. Cell 54: $755-764$.

Galjart, N.J., Gillemans, N., Meijer, D. and d'Azzo, A. 1990. Mouse "protective protein": cDNA cloning, sequence comparison, and expression. J. Biol. Chem. 265: 4678-4684.

Hoogeveen, A.T., Verheijen, F.W. and Galjaard, H. 1983. The relation between human lysosomal $\beta$-galactosidase and its protective protein. J. Biol. Chem. 258: 12143-12146.

Nanba, E., Tsuji, A., Omura, K. and Suzuki, Y. 1987. Galactosialidosis: direct evidence that a 46-kilodalton protein restores deficient enzyme activities in fibroblasts. Biochem. Biophys. Res. Commun. 144: 138-142.

Potier, M., Michaud, L., Tranchemontagne, J. and Thaurette, L. 1990. Structure of the lysosomal neuraminidase- $\beta$-galactosidase-carboxypeptidase multienzymic complex. Biochem. $J$. 267: 197-202.

Sakuraba, H., Aoyagi, T. and Suzuki, Y. 1982. Galactosialidosis ( $\beta$-galactosidase-neuraminidase deficiency): a possible role of serine-thiol proteases in the degradation of $\beta$-galactosidase molecules. Clin. Chim. Acta 125: 275-282.

Stevens, T.H., Rothman, J.H., Payne, G.S. and Schekman, R. 1986. Gene dosage-dependent secretion of yeast vacuolar carboxypeptidase Y. J. Cell Biol. 102: 1551-1557.

Suzuki, Y., Nanba, E., Tsuji, A., Yang, R.-C., Okamura-Oho, Y. and Yamanaka, T. 1988. Clinical and genetic heterogeneity in galactosialidosis. Brain Dysfunct. 1: 285-293.

Suzuki, Y., Sakuraba, H., Yamanaka, T., Ko, Y.-K., Iimori, Y., Okamura, Y. and Hoogeveen, A.T. 1984. Galactosialidosis: A comparative study of clinical and biochemical data on 22 patients. In The Developing Brain and Its Disorders, Arima, M., Suzuki, Y. and Yabuuchi, H., eds., Univ. Tokyo Press, Tokyo, pp. 161-175.

Tranchemontagne, J., Michaud, L. and Potier, M. 1990. Deficient lysosomal carboxypeptidase activity in galactosialidosis. Biochem. Biophys. Res. Commun. 168: 22-29.

Tsuji, S., Yamada, T., Ariga, T., Toyoshima, I., Yamaguchi, H., Kitahara, Y. and Miyatake, T. 1983. Carrier detection of sialidosis with partial $\beta$-galactosidase deficiency by the assay of lysosomal sialidase in lymphocytes. Ann. Neurol. 15: 181-183.

Verheijen, F.W., Palmeri, S., Hoogeveen, A.T. and Galjaard, H. 1985. Human placental neuraminidase. Activation, stabilization and association with $\beta$-galactosidase and itsprote ctive protein. Eur. J. Biochem. 149: 315-321. 